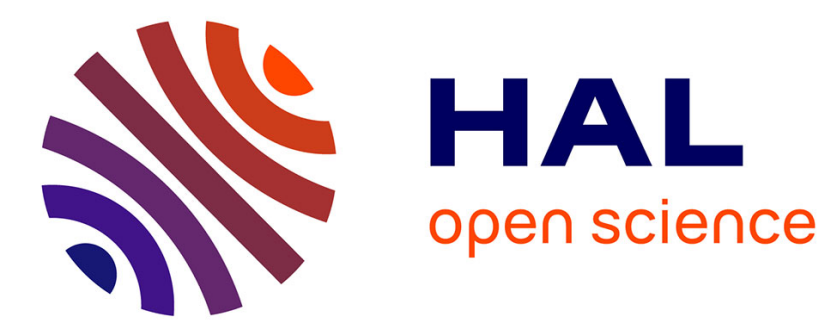

\title{
Uncertainty propagation methods in dioxin/furans emission estimation models
}

G. Ripamonti, G. Lonati, Piero Baraldi, F. Cadini, Enrico Zio

\section{To cite this version:}

G. Ripamonti, G. Lonati, Piero Baraldi, F. Cadini, Enrico Zio. Uncertainty propagation methods in dioxin/furans emission estimation models. ESREL 2011, Sep 2011, Troyes, France. pp.2222 - 2229. hal-00658104

\section{HAL Id: hal-00658104 \\ https://hal-centralesupelec.archives-ouvertes.fr/hal-00658104}

Submitted on 12 Jan 2012

HAL is a multi-disciplinary open access archive for the deposit and dissemination of scientific research documents, whether they are published or not. The documents may come from teaching and research institutions in France or abroad, or from public or private research centers.
L'archive ouverte pluridisciplinaire HAL, est destinée au dépôt et à la diffusion de documents scientifiques de niveau recherche, publiés ou non, émanant des établissements d'enseignement et de recherche français ou étrangers, des laboratoires publics ou privés. 


\title{
Uncertainty propagation methods in dioxin/furans emission estimation models
}

\author{
G. Ripamonti \& G. Lonati \\ DIIAR-Environmental Section - Politecnico di Milano, Milano, Italy
}

P. Baraldi, F. Cadini,

Dipartimento di Energia - Politecnico di Milano, Milano, Italy

E. Zio

Ecole Centrale Paris and Supelec, Paris, France \& Dipartimento di Energia - Politecnico di Milano, Milano, Italy

ABSTRACT: In this paper we propose a comparison between two different approaches for uncertainty propagation in Environmental Impact Assessment (EIA) procedures. Both a purely Probabilistic (PMC) and a Hybrid probabilistic-possibilistic Monte Carlo method (HMC) are applied on an estimation model of dioxin/furans emission from a waste gasification plant. The analysis shows that when input variables affected by scarcity of information are present, HMC seems to be a valid alternative method that properly propagates uncertainty from data to output avoiding arbitrary and subjective assumptions on the input probability distribution functions. HMC could improve the transparency of the EIA procedure with positive effects on the communicability and credibility of its predictions.

\section{INTRODUCTION}

According to the current EU regulations (Directive 85/337/EEC as amended by Directives 97/11/EC and 2003/35/EC), an Environmental Impact Assessment (EIA) is required for public and private projects likely to have significant impacts on the environment by virtue inter alia of their nature, size or location. The scope of an EIA is the identification, description and assessment of project's direct and indirect effects on human beings, fauna and flora, soil, water, air, climate, landscape, material assets and cultural heritage. EIA procedure is a part of the decisional process for the approval of a proposed project. Predictions of EIA help and orient the activity of decision makers ensuring that environmental considerations are taken into account. Furthermore, EIA serves an important procedural role in the overall decision-making process by promoting public information and participation. Therefore, an EIA study needs to be carried out considering that its output will be not only handled by expertise but also it will be communicated to decision maker, stakeholders and local population. Assuming that in most cases the proposed project does not meet popular consensus, communicability of EIA predictions to residents and stakeholders plays a major and challenging role. To this purpose the whole EIA procedure must be an open and transparent process leading to robust and reproducible outputs for informed decision-making and correct public awareness.
In this context, waste disposal installations for the incineration of both hazardous and non-hazardous waste are subject to EIA, in particular with regards to their potential impact on air quality.

With reference to the proposal of a new project, EIA consists of three main steps (Figure 1):

a) the characterization of the source: estimation of the atmospheric emissions from the operation of the plant in terms of both the types and the quantities of pollutants;

b) the estimation of the atmospheric pollutant concentration levels in the area close to the proposed new installation by means of proper dispersion models;

c) the assessment of the pollutant concentration levels at the receptor, resulting from the superposition of the estimated concentration to the existing background concentration levels

The first objective is that of proving the compliance of the estimated concentrations with both long-term (i.e.: annual average) and short-term (i.e.: hourly and daily average) air quality limits. Secondly, as those installations are responsible for the emission of metals, dioxins and furans (PCDD/Fs), additional evaluations may also include human health risk assessment for both carcinogenic and noncarcinogenic pollutants. This latter assessment is characterized by two main features: i) a considerable complexity of the mechanisms involved and hence related uncertainties, ii) a general attitude of "aversion to risk", with implications on the way uncertainties are treated (Guyonnet et al., 2008). 


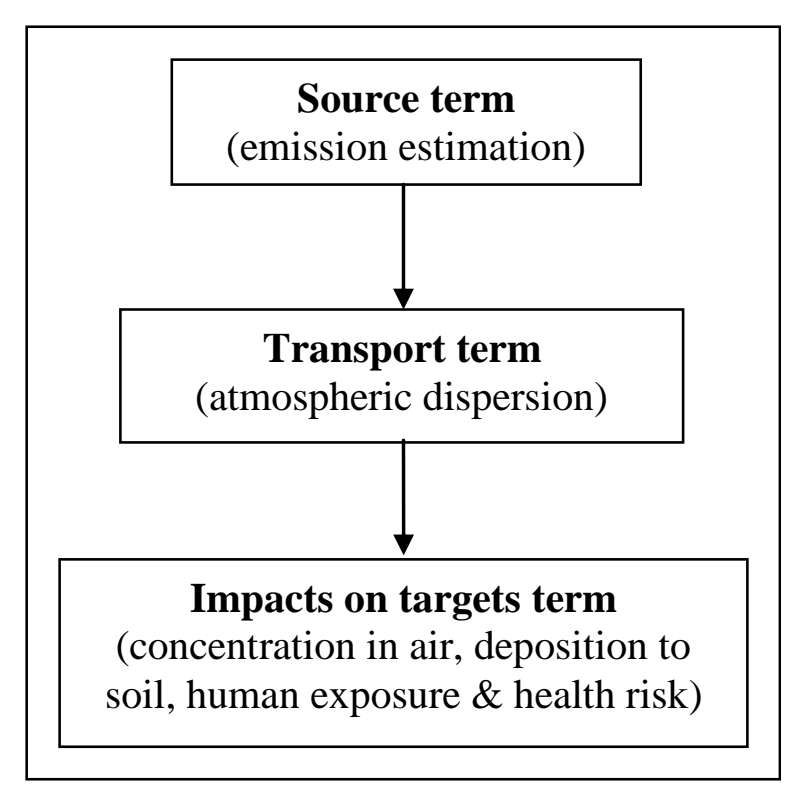

Figure 1 Schematic framework of the main steps of the EIA for industrial plants.

These uncertainties, which affect all the three steps of the EIA procedure, may be originated from randomness of process or from scarcity of information and lack of knowledge. In particular, regardless for the approaches and models adopted to estimate the plant emissions and their dispersion in the atmosphere, the final concentrations and deposition estimates are seriously affected by the uncertainty of the values of the source term parameters.

The attitude of "aversion to risk" often leads to answer to the question whether a given "acceptable" threshold may be exceeded relying on limiting scenario calculations. In common practice, this means that input parameter uncertainties are often neglected and a simplistic deterministic approach is adopted, in which the emissions are conservatively estimated on the basis of project design data and assuming i) a full-load plant operation and ii) the emission concentrations of the pollutants equal to the emission regulatory limits. This approach is often excessively conservative, and does not provide a realistic and objective description of the plant impact on the environment.

To overcome this problem, the EIA procedures can be applied within a probabilistic approach for i) describing the uncertainties of the input parameters and ii) propagating them to the models outputs, i.e. the atmospheric pollutant concentrations. In the probabilistic approach the uncertain variables are described by means of probability distribution functions (PDFs), which are then propagated through the impact assessment model by Monte-Carlo (MC) simulation (Lonati et al. 2007) (Schuhmacher et al. 2001, Sonnemann et al. 2002).

However, in general, the construction of the empirical PDFs representing the uncertainty in the input parameters by statistical analysis may not be an easy task due to the scarce data availability typical of the environmental context (Ferson \& Ginzburg 1996, Baudrit et al. 2006). Within a probabilistic framework the analyst solves this task forcing a PDF on the scarce available data on the basis of his subjective judgement. This operation presents two important drawbacks:

- doubts have been raised on the consistency of this forced PDF with the incomplete/imprecise information at hand. The risk is to introduce information that is in fact not available.

- this subjective and arbitrary view of probability adds assumptions in the analysis, which are often neither declared nor justified, causing a loss of transparency in the procedure.

These drawbacks are of particular relevance, considering the EIA role as decision aiding tool and informative tool. A lack of transparency in the procedure can lead to a loss of credibility among decision makers and public.

A number of alternative representations of uncertainty under limited information availability (e.g.: fuzzy set theory, evidence theory, possibility theory and interval analysis) have been proposed and applied in different field of risk assessment (Klir \& Yuan 1995; Aven \& Zio 2011). Among them, Possibility Theory has received growing attention because of its representation power and its relative mathematical simplicity. In recent past years some applications of these alternative approaches have also been exploited in the environmental risk assessment field (Kumar et al. 2009, Guyonnet et al. 2003, Kentel \& Aral 2005, Li et al. 2007).

In practice, different input parameters of the same model may be characterized by different amounts of information availability, thus possibly giving rise to both probabilistic and possibilistic uncertainty representations. In this regard, recently Hybrid probabilistic-possibilistic Monte Carlo methods (HMC) have been proposed for combining both representations in the same uncertainty propagation analysis (Baudrit et al. 2006, Baraldi \& Zio, 2008, Flage et al. 2010).

In this work a HMC method is applied to an uncertainty propagation analysis in an emission estimation model for PCDD/Fs in a planned new waste gasification plant. The results are compared to those obtained by a standard PMC approach, showing a better capability of handling limited data knowledge, without forcing statistically unjustified probability distributions on data. The positive aspect of this approach with respect to the transparency of the EIA procedure and to the communicability of results are also discussed.

The paper is organized as follows. In Section 2 the HMC and PMC methods are methodologically compared on a case study regarding the emission model of dioxin/furans from a waste gasification plant; the results are provided in Section 3. Finally, some conclusions on the advantages of the HMC method are 
drawn in Section 4. In the appendix Section A1 provides the basics of possibility theory and Section A2 presents the pure Probabilistic Monte Carlo method for uncertainty propagation and its evolution to the Hybrid probabilistic-possibilistic Monte Carlo method under scarce data availability.

\section{CASE STUDY: THE EMISSION MODEL OF DIOXIN/FURANS FROM A WASTE GASIFICATION PLANT}

The uncertainty representation and propagation methods introduced in Section 3 are applied to the first step of the EIA procedure with reference to a planned waste gasification plant. The objective is the estimation of dioxin/furan (PCDD/F) emission within the flue gas released at the stack of the plant. Notice that $\mathrm{PCDD} / \mathrm{F}$ pollutants have a relevant role in health risk assessment due to their carcinogenic potential and their persistency in the environment, once released into the atmosphere.

The emission model adopted computes the emitted PCDD/F mass flow, $Q(\mathrm{ng} / \mathrm{s})$, as a function of the plant daily throughput, $P\left(\mathrm{Mg}_{\text {waste }} /\right.$ day $)$, the $\mathrm{PCDD} / \mathrm{F}$ concentration in the emitted flue gas, $C_{D}\left(\mathrm{ng} / \mathrm{m}^{3}\right)$, and the specific gas production $V_{F}\left(\mathrm{~m}^{3} / \mathrm{Mg}_{\text {waste }}\right)$ :

$$
Q=\frac{P \cdot C_{D} \cdot V_{F}}{3600 \cdot 24}
$$

As usual for this kind of evaluations, the PCDD/F concentration is expressed in terms of equivalent toxicity mass per unit volume at normal conditions $\left(0^{\circ} \mathrm{C}, 101.3 \mathrm{kPa}\right)$; coherently, the specific gas production refers to the same temperature and pressure conditions.

The input parameters can be characterized as follows:

- the plant daily throughput $P$ is a constant parameter whose value is assigned during project design. In the plant of the case study, $P$ is set to the value of $9000 \mathrm{Mg}_{\text {waste }} /$ day.

- the PCDD/F concentration $C_{D}$ is an uncertain parameter whose value varies during normal gasification operation due to the fluctuations of the process parameters and to the heterogeneous and variable composition of the fed waste.

- the specific gas production $V_{F}$ is an uncertain parameter. Although its value is set during the project design phase, $V_{F}$ can present variations during plant operation caused by fluctuations of the energy content of the fed waste, which is characterized by an heterogeneous and variable composition.
Due to the rather limited applications of the waste gasification process, datasets containing $C_{D}$ e $V_{F}$ values collected during operation of similar plants are not available. However, different studies have recently investigated the pollutant emission from waste gasification plants (University of California 2009, Yamada et al. 2004, Porteous 2005, Arena et al. 2008b, Klein 2002). From these studies, it has been possible to identify $35 C_{D}$ and $4 V_{F}$ values.

Given the availability of a statistically significant set of $C_{D}$ values, the uncertainty on this model parameter has been represented using a probability distribution. A Kolmogorov-Smirnov's test considering lognormal, Weibull, Beta, logistic PDFs has been performed in order to properly choose the PDF representative of the available data. Figure 1 shows the selected Beta-PDF of parameters $\alpha=0.36$ and $\beta=$ 1.32 and maximum value $0.07 \mathrm{ng} / \mathrm{m}^{3}$.

With respect to $V_{F}$, the available information (four literature values and the case study project value) is very scarce. Thus, two different alternative representations of the uncertainty affecting $V_{F}$ have been considered:

- a subjective triangular PDF based on analyst judgment. The PDF range is [3360, 6670] corresponding to the minimum and maximum values of the four available literature values and its mode is 5420 corresponding to the current case study project value (Figure 2).

- a triangular possibility distribution with the same range of the subjective PDF and most likely value set equal to the case study project value (Figure $3)$.

Notice that, although the possibility and probability distributions used to represent the uncertainty on $V_{F}$ have similar shapes, they convey very different information: the possibility distribution summarizes a set of cumulative distributions, bounded by the so-called Necessity and Possibility functions, whereas the subjective PDF defines just one specific cumulative distribution of that set.

Table 1 summarizes the different distributions used in this work for the representation of the uncertainty on the two input parameters. With respect to the uncertainty propagation, the PMC method is applied to the case in which the uncertainty on $C_{D}$ and $V_{F}$ are represented by the PDFs, whereas the HMC method considers the PDF for $C_{D}$ and the possibility distribution for $V_{F}$.

The number $m$ of MC realizations of the parameters $C_{D}$ and $V_{F}$ used in the PMC method has been set to 1000 . The HMC procedure, instead, has been run considering $m=1000$ realizations of the parameter $C_{D}$ and, for each of these realizations, $21 \alpha$ values 
(range 0-1, step 0.05) for the possibilistic variable $V_{F}$.

Although $\mathrm{C}_{\mathrm{D}}$ and $\mathrm{V}_{\mathrm{F}}$ are expected to be somehow correlated, both PMC and HMC methods have been applied assuming independency between the two parameters. Notice that, due to the complexity of the waste gasification process and to the high number of involved parameters, information useful to model the dependence between $\mathrm{C}_{\mathrm{D}}$ and $\mathrm{V}_{\mathrm{F}}$ has not been found in literature.

Table 1 Distributions used to represent the uncertainty on the model parameters by the PMC and HMC method

\begin{tabular}{lccc}
\hline Parameter & Type & PMC method & HMC method \\
\hline$P$ & Constant & 9000 & 9000 \\
$C_{D}$ & Uncertain & \multicolumn{2}{c}{ Beta-PDF } \\
& & {$[\alpha=0.36, \beta=1.22$, Max $=0.07]$} \\
$\mathrm{V}_{\mathrm{F}}$ & Uncertain & $\begin{array}{c}\text { Triangular-PDF } \\
{[3360,6670,5420]}\end{array}$ \\
\hline
\end{tabular}

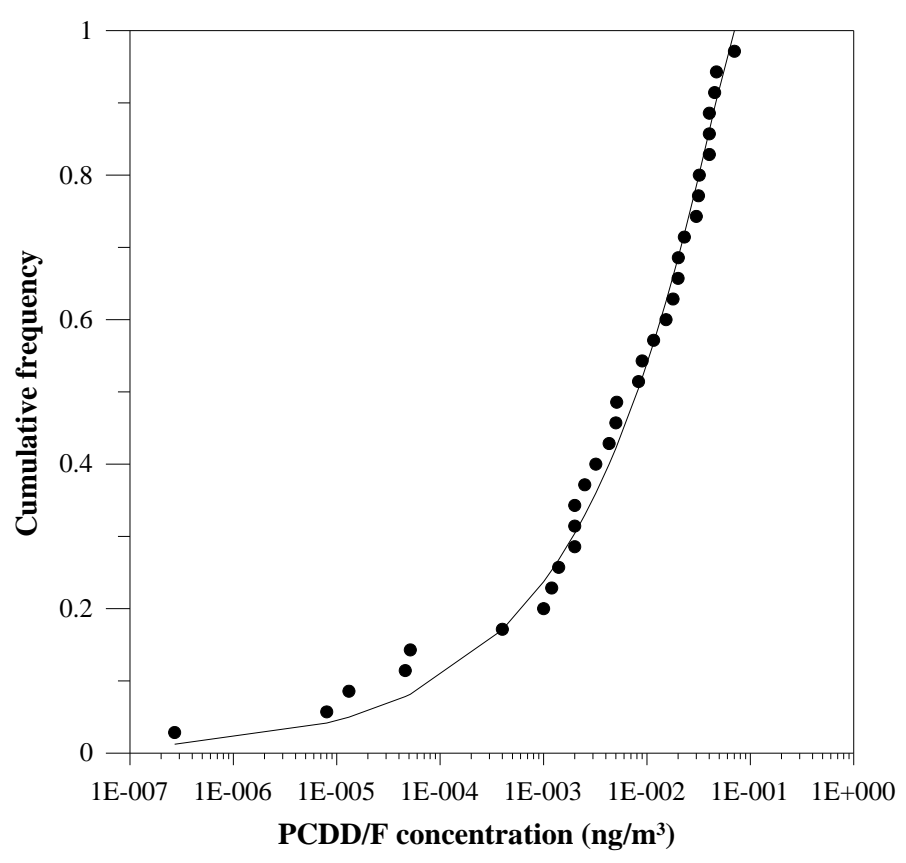

Figure 2 Empirical cumulative distribution (dots) of the data used to estimate the PCDD/F concentration, $\mathrm{C}_{\mathrm{D}}$, and Beta distribution used to fit the data.

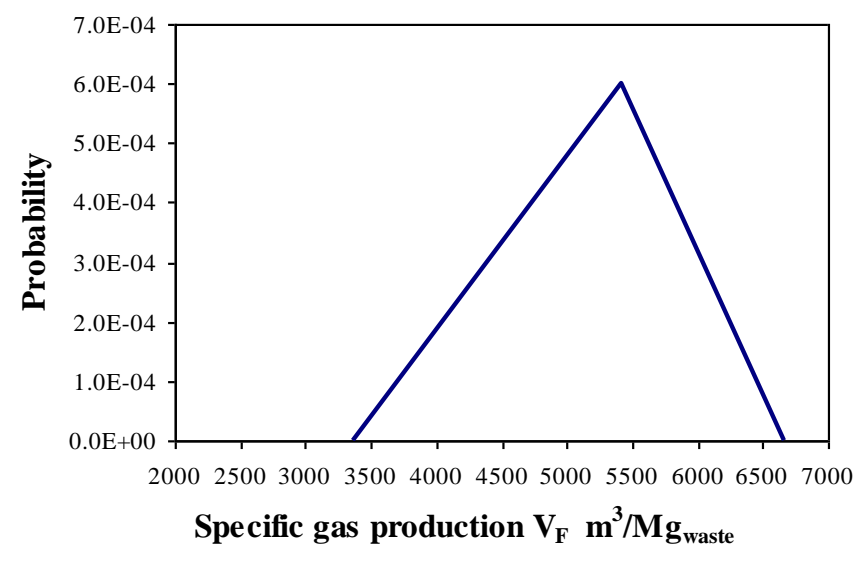

Figure 3 Subjective triangular-PDF of the specific gas production, $V_{F}$, considered by the PMC method

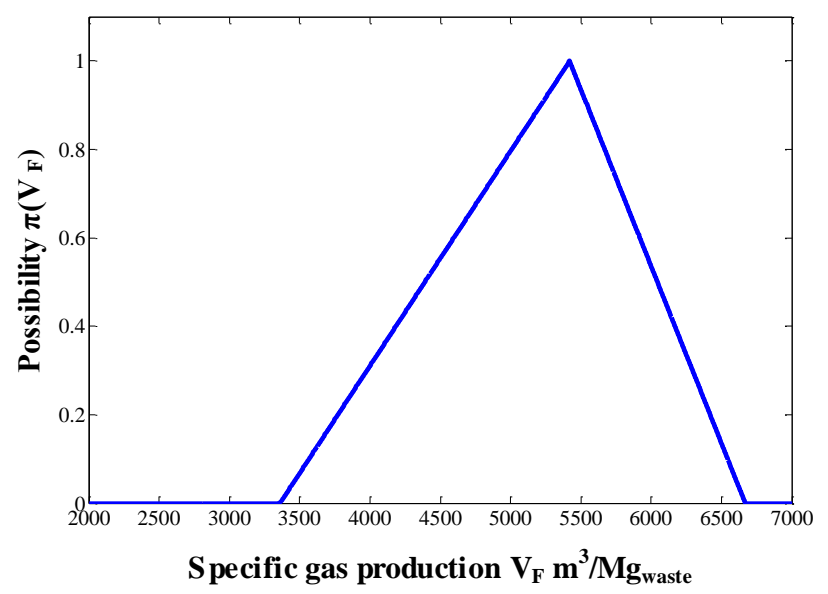

Figure 4 Triangular possibility distribution of the specific gas production, $V_{F}$, considered by the HMC method

\section{RESULTS}

The cumulative distributions of the model output variable, $Q$, obtained by applying the PMC and HMC uncertainty propagation methods are presented in Figure 5. The PMC method provides a single cumulative distribution (continuous line), whereas the HMC model provides the belief (lower dotted curve) and the plausibility (upper dashed curve) distributions, which can be interpreted as the boundary cumulative distributions of $Q$.

As expected, the cumulative distribution of the model output obtained by the PMC is within the belief and plausibility functions obtained by the hybrid approach.

Notice that the representation of the uncertainty on $Q$ provided by the PMC method is more concise and easy to be interpreted than that provided by the HMC method. However, this is a consequence of the different representation of the uncertainty on the input parameter $V_{F}$ : while the HMC method considers a set of cumulative distributions, the PMC forces all the uncertainty on $V_{F}$ to be represented by a single 
distribution. Given the scarcity of information available in this case, the use of a PDF seems statistically unjustified.

It can also be seen that the HMC method allows to process separately the uncertainty on $C_{D}$, represented by a probability distribution, from the uncertainty on $V_{F}$, represented by a possibility distribution. These two contributions are explicitly visible in the results: the uncertainty on $Q$ affects the slope of the belief and plausibility functions, while the separation between the belief and plausibility functions reflects the imprecision in the knowledge of the parameter $V_{F}$. In the PMC method, instead, the contributions of the uncertainty on the two input parameters onto the output uncertainty are merged and result in the slope of the output cumulative distribution.

Within the EIA procedure it can be interesting to lump all the information contained in the obtained cumulative distribution of $Q$ into a single value such as a $\beta$ percentile. To this purpose, setting a degree of confidence $\beta=0.95$, the PMC method provides a single value, $Q^{95}=3.08 \mathrm{ng} / \mathrm{s}$, representative of the $95^{\text {th }}$ percentile. Using the HMC, the $95^{\text {th }}$ percentile is an uncertain quantity whose true value is in the interval $[2.52 \mathrm{ng} / \mathrm{s}-3.42 \mathrm{ng} / \mathrm{s}]$ corresponding to the $95^{\text {th }}$ percentile of the Belief and Plausibility functions. Table 2 reports the values of different percentiles both for PMC and HMC methods, as visible PMC values are always in the range of HMC values and the distance between the plausibility and belief functions increases from lower to higher percentile.

Finally, notice that these estimates of the $95^{\text {th }}$ percentile of the emitted PCDD/F mass flow, $Q$, obtained with both uncertainty propagation methods are one order of magnitude lower than the estimate, $Q=56.4 \mathrm{ng} / \mathrm{s}$, calculated with a deterministic approach based on the plant design data and on the $\mathrm{PCDD} / \mathrm{F}$ regulatory emission limit $\left(0.1 \mathrm{ng} / \mathrm{m}^{3}\right)$.

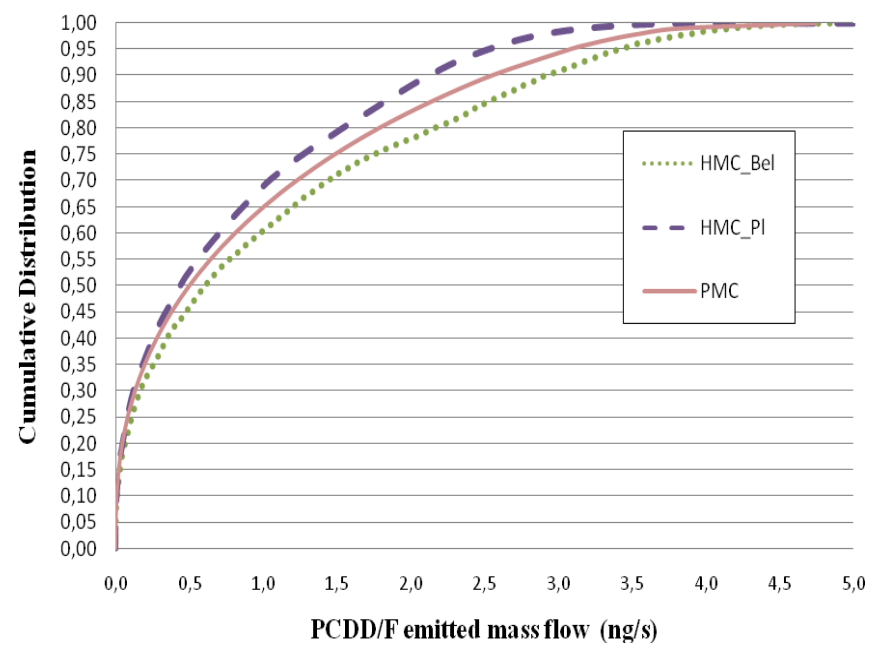

Figure 5 Comparison of the cumulative distributions of the emitted PCDD/F mass flow (ng/s), $Q$, obtained by the PMC and HMC methods.
Table 2 Estimated PCDD/F mass flow (ng/s): comparison of some percentiles obtained by the PMC and HMC method.

percentile

\begin{tabular}{lccc}
\hline Method & $\mathbf{0 . 5 0}$ & $\mathbf{0 . 7 5}$ & $\mathbf{0 . 9 5}$ \\
\hline $\begin{array}{l}\text { PMC } \\
\text { Probabilistic Monte-Carlo }\end{array}$ & 0.50 & 1.49 & 3.08 \\
$\begin{array}{l}\text { HMC } \\
\text { Hybrid Monte-Carlo }\end{array}$ & {$[0.44,0.61]$} & {$[1.27,1.75]$} & {$[2.52,3.42]$} \\
\hline
\end{tabular}

\section{CONCLUSION}

In this paper a Hybrid probabilistic and possibilistic Monte Carlo method (HMC) has been applied to the Dioxin/Furan emission assessment model of a planned waste gasification plant and the results have been compared to those obtained by a pure Probabilistic Monte-Carlo method (PMC).

When input variables affected by scarcity of information are present, the HMC method has shown to be more effective in propagating the input uncertainties through the model in that i) the information provided by its outputs is more consistent with that available for the input parameters and ii) uncertainty is processed more "transparently" than with PMC methods, thus avoiding arbitrary and subjective assumptions by the analyst on the input probability distribution functions. Moreover, the HMC method allows separating the contributions to the output uncertainty due to the probabilistic and the possibilistic input parameters.

This work has been conceived as a preliminary study to understand the applicability of HMC methods to the complete EIA procedure. In this regard, the satisfactory outcomes of this first analysis foster future works towards the extension of the HMC method to the remaining stages of an EIA procedure.

Finally, it is worth noticing that the principal users of EIA outputs are decision-makers often responsible for communicating the results to the population living in the area of the planned installation. With respect to environmental and health-related issues, there is in general social aversion to accept information expressed in terms of probability. Therefore, future studies will also have to investigate the way of post-processing the results of a whole EIA procedure developed within an hybrid probabilisticpossibilistic framework to make them communicable to the stakeholders and to make them more easily understandable. To this purpose, the abilities of the HMC method to avoid arbitrary assumptions and to provide results that explicitly report both probabilistic and possibilistic uncertainties could be useful for a transparent and clear post-process. 


\section{A.1. BASIC OF POSSIBILITY THEORY}

In possibility theory, uncertainty is represented by a possibility function $\pi(y)$. For each $y$ in a set $\Omega$, $\pi(y)$ expresses the degree of possibility of $y$. When $\pi(y)=0$ for some $y$, it means that the outcome $y$ is considered an impossible situation. When $\pi(y)=1$ for some $y$, it means that the outcome $y$ is possible, i.e.: is just unsurprising, normal, usual (Dubois 2006). This is a much weaker statement than when probability is 1 .

The possibility function gives rise to probability bounds, upper and lower probabilities, referred to as necessity and possibility measures $(N, \Pi)$. The possibility of an event $A, \Pi(A)$, is defined by

$$
\Pi(A)=\sup _{y \in A}\{\pi(y)\},
$$

and the necessity measure $N(A)$ is defined by

$$
N(A)=1-\Pi(\text { not } A)=1-\sup _{y \notin A}\{\pi(y)\} .
$$

Let $\mathcal{P}(\pi)$ be a family of probability distributions such that for all events $A, N(A) \leq P(A) \leq \Pi(A)$. Then,

$$
N(A)=\inf P(A) \text { and } \Pi(A)=\sup P(A)
$$

where inf and sup are with respect to all probability measures in $\mathcal{P}$. Hence the necessity measure is interpreted as a lower level for the probability and the possibility measure is interpreted as an upper limit. Referring to subjective probabilities, the bounds reflect that the analyst is not able or willing to precisely assign his/her probability, and the bounds are the best he/she can do given the information available; in other words, he or she can only describe a subset of $\mathcal{P}$ which contains his/her probability (Dubois 2006).

\section{A.2. PROBABILISTIC-POSSIBILISTIC UNCERTAINTY REPRESENTATION AND PROPAGATION THROUGH A MODEL}

Let us consider a model whose output $Z=f\left(Y_{1}, Y_{2}, \ldots, Y_{n}\right)$ is a function of $n$ uncertain variables $Y_{i}, i=1, \cdots, n$. The uncertainties of the first $\mathrm{k}$ variables can be represented by probability distributions $p_{Y_{i}}(y)$ properly derived from data, whereas for the remaining $n-k$ the scarcity of information provide a weaker statistical base for a specific probability assignment, making the construction of PDFs a critical operation. Two different approaches are here presented to represent and propagate such uncertainties
The uncertainties of all $n$ input variables are treated in terms of probability distributions. Probability representations of the $n-k$ variables can be assigned i.e.: based on expert judgment. For example, let us considered a typical case in which the available information on a model parameter $x$ is only that its values are located somewhere between a value $x_{\text {min }}$ and a value $x_{\max }$. In this case, a uniform probability distribution $p(x)=1 /\left(x_{\max }-x_{\min }\right), \forall x \in\left[x_{\min }, x_{\max }\right]$ is typically assumed to represent the uncertainty on $x$. This approach appeals to: i) Laplace principle of insufficient reason according to which all that is equally plausible is equally probable and to ii) the maximum entropy approach (Baraldi et al. 2010). However, doubts on the consistency of this uncertainty representation have been raised (Baudrit et al. 2006) as it seems that the insufficient knowledge may justify choices of specific functional probability distributions, like the uniform, but it should somehow account for the full set of possible probability distributions on $\mathrm{X}=\left|x_{\min }, x_{\max }\right|$, so that the probability of value $x \in \mathrm{X}$ is allowed to take any values in |0,1| (Baraldi et al. 2010).

The PMC method then applies a single loop Monte Carlo simulation to propagate the uncertainties of the $n$ variables through the model. The operative steps of the propagation procedure are the following:

1 Sample the $i$-th realization $\left(y_{1}^{i}, \cdots, y_{n}^{i}\right)$ of the uncertain variables $\left(Y_{1}, \cdots, Y_{n}\right)$ from their respective PDFs $p_{Y_{1}}\left(y_{1}\right), \ldots, p_{Y_{n}}\left(y_{n}\right)$

2 Compute the output of the model corresponding to the $i-t h$ realization of the uncertain variables: $\mathrm{Z}_{i}=f\left(y_{1}^{i}, \ldots ., y^{i}{ }_{n}\right)$

3 Repeat steps 1 and 2 for many times. Then, derive the cumulative distribution $F(Z)$ from the outputs $\mathrm{Z}_{i}, i=1, \ldots, m$

The cumulative distribution $F(Z)$ obtained gives information on the output uncertainty. Setting a percentile $\beta$, that corresponds to the desired degree of confidence, a concise output value of $Z$ can be provided. The output realizations $\mathrm{Z}_{i}, i=1, \ldots, m$, found in step 2 can be directly used to compute other lumped indicators of the output distribution, such as its mean and standard deviation values.

\section{b. Hybrid probabilistic-possibilistic Monte-Carlo method (HMC)}

In the Hybrid probabilistic-possibilistic method, the uncertainties of the $n-k$ variables are represented in terms of possibility distributions $\left(\pi^{Y_{k+1}}, \cdots, \pi^{Y_{n}}\right)$. Different methods have been developed to derive possibility distributions from the available information (Baudrit \& Dubois 2006, Dubois 1993). Often the analyst only knows that an uncertain variable can take values in a given range $[a, b]$ wherein the most 
likely value is $c$. Triangular possibility function with the range $|a, b|$ taken as base and $c$ taken as vertex can be typically used to describe this information. It has been shown that the family of probability distributions defined by such possibility distribution with contains all the probability distributions with support $[a, b]$ and mode $c$ (Baudrit \& Dubois 2006).

In the HMC method, the propagation of the uncertainties expressed in terms of both PDFs and possibility curves is performed by combining the Monte Carlo technique with fuzzy interval analysis (Baudrit et al. 2006). The operative steps of the propagation procedure are the following:

1 sample the $i$-th realization $\left(y_{1}^{i}, \cdots, y_{k}^{i}\right)$ of the random variable vector $\left(Y_{1}, \cdots, Y_{k}\right)$

2 select a possibility value $\alpha \in[0,1]$ and the corresponding $\alpha$ - cuts of the possibility distributions $\left(\pi^{Y_{k+1}}, \cdots, \pi^{Y_{n}}\right)$, i.e: the intervals of values of the possibilistic variables $\left(Y_{k+1}, \cdots, Y_{n}\right)$ with associated possibility distributions greater or equal to $\alpha$.

3 calculate the smallest and largest values of $f\left(y_{1}^{i}, \cdots, y_{k}^{i}, Y_{k+1}, \cdots, Y_{n}\right)$, denoted by $f^{i}$ and $\bar{f}_{\alpha}^{i}$ respectively, considering the fixed values $\left(y_{1}^{i}, \cdots, y_{k}^{i}\right)$ sampled in 1 . for the random variables $\left(Y_{1}, \cdots, Y_{k}\right)$ and all values of the possibilistic variables $\left(Y_{k+1}, \cdots, Y_{n}\right)$ in the $\alpha$ - cuts of their possibility distributions $\left(\pi^{Y_{k+1}}, \cdots, \pi^{Y_{n}}\right)$ found in 2. Then, consider the extreme values $f^{i}$ and $\bar{f}_{\alpha}^{i}$ found in 3. as the lower and upper limit of the $\alpha$-cut of $f\left(y_{1}^{i}, \cdots, y_{k}^{i}, Y_{k+1}, \cdots, Y_{n}\right)$

4 return to step 2. and repeat for another $\alpha$ - cut; the fuzzy random realization (fuzzy interval) $\pi_{i}^{f}$ of $Z=f(Y)$ is obtained as the collection of the values $f_{\alpha}^{i}$ and $\bar{f}_{\alpha}^{i}$ for each $\alpha-c u t$

5 return to step 1 . to generate a new realization of the random variables.

The procedure is repeated for $i=1, \cdots, m$ : at the end of the procedure $m$ realizations of fuzzy intervals are obtained, i.e.: $\left(\pi_{1}^{f}, \cdots, \pi_{m}^{f}\right)$.

For each set $A$ contained in the universe of discourse $U_{Z}$ of the output variable $Z$, it is possible to obtain the possibility measure $\Pi_{i}^{f}(A)$ and the necessity measure $N_{i}^{f}(A)$ from the corresponding possibility distribution $\pi_{i}^{f}(z)$, by:

$$
\begin{aligned}
& \Pi_{i}^{f}(A)=\max _{z \in A}\left\{\pi_{i}^{f}(z)\right\} \\
& N_{i}^{f}(A)=\inf _{z \notin A}\left\{1-\pi_{i}^{f}(z)\right\}=1-\Pi_{i}^{f}(\bar{A}) \\
& \forall A \subseteq U_{Z}
\end{aligned}
$$

Finally, the $m$ different realizations of possibility and necessity measures can be combined to obtain the belief $\operatorname{Bel}(A)$ and the plausibility $\operatorname{Pl}(A)$ for any set $A$, respectively (Baudrit et al. 2006):

$$
\begin{aligned}
& \operatorname{Bel}(A)=\sum_{i=1}^{m} \frac{N_{i}^{f}(A)}{m} \\
& \operatorname{Pl}(A)=\sum_{i=1}^{m} \frac{\prod_{i}^{f}(A)}{m}
\end{aligned}
$$

For each set $A$, this technique computes the belief and plausibility as the average of the possibility measures associated with each output fuzzy interval.

The likelihood of the value $f(Y)$ passing a given threshold $z$ can then be computed by considering the belief and the plausibility of the set $A=(-\infty, z]$; in this respect, $\operatorname{Bel}(f(Y) \in(-\infty, z])$ and $\operatorname{Pl}(f(Y) \in$ $(-\infty, z])$ can be interpreted as bounding, average cumulative distributions $\underline{F}(z)=\operatorname{Bel}(f(Y) \in(-\infty, z])$, $\bar{F}(z)=P l(f(Y) \in(-\infty, z]) \quad$ (Baudrit et al. 2006). Thus, one way to estimate the total uncertainty on $f(Y)$ is to provide a confidence interval at a given level of confidence, taking the lower and upper bounds from $P l(f(Y) \in(-\infty, z])$ and $\operatorname{Bel}(f(Y) \in$ $(-\infty, z])$, respectively (Baudrit et al. 2006). Notice, however, that it is not possible to directly obtain information on the mean and standard deviation of the output distribution from the plausibility and belief distributions.

\section{REFERENCES}

Arena, U., Zaccariello, L., Mastellone, M.L. 2008b. Gasification of a plastic waste in a fluidized bed of olivine. In: Werther, J., Nowak, W., Wirth, K.-E., Hartge, E.-U. (Eds.), Proceedings of CFB9 - 9th International Conference on Circulating Fluidized Beds : 691-696, ISBN 978-3930400-57-7.

Aven, T. \& Zio, E. 2011. Some considerations on the treatment of uncertainties in risk assessment for practical decision making. Reliability Engineering and System Safety, 96 (1): 64-74.

Baraldi, P. \& Zio, E. 2008. A Combined Monte Carlo and Possibilistic Approach to Uncertainty Propagation in Event Tree Analysis. Risk Analysis, 28 (5): 1309-1326.

Baraldi, P., Popescu, I.C., Zio, E. 2010. Methods of Uncertainty Analysis in prognostics. International Journal of Perfonance Engineering, 6 (4): 305-326

Baudrit, C. \& Dubois, D. 2006. Practical Representations of Incomplete Probabilistic Knowledge. Computational Statistics \& Data Analysis, 51 (1): 86-108.

Baudrit, C., Dubois, D., \& Guyonnet, D. 2006. Joint Propagation of Probabilistic and Possibilistic Information in Risk Assessment. IEEE Transactions on Fuzzy Systems, 14 (5): 593-608.

Dubois, D. 2006. Possibility theory and statistical reasoning. Computational Statistics \& Data Analysis, 51 (1): 47-69

European Union Council Directive 85/337/EEC of 27 June 1985 on the assessment of the effects of certain public and private projects on the environment. Official Journal L 175, 5.7.1985.

European Union Council Directive 97/11/EC of 3 March 1997 amending Directive 85/337/EEC on the assessment of the 
effects of certain public and private projects on the environment. Official Journal L 73, 14.3.1997.

European Union Directive 2003/35/EC of the European Parliament and of the Council of 26 May 2003, providing for public participation in respect of the drawing up of certain plans and programmes relating to the environment and amending with regard to public participation and access to justice Council Directives 85/337/EEC and 96/61/EC. Official Journal L 156, 25.6.2003.

Ferson, S. \& Ginzburg, L. 1996. Different methods are needed to propagate ignorance and variability. Reliability Engineering and system safety 54:133-144.

Flage, R., Baraldi, P., Zio, E., Aven, T. 2010. Possibilityprobability transformation in comparing different approaches to the treatment of epistemic uncertainties in a fault tree analysis. In B. Ale, I.A. Papazoglu, E. Zio (Eds.), Reliability, Risk and Safety - Proceedings of the European Safety and RELiability (ESREL) 2010 Conference, Rhodes, Greece: 714-721.

Guyonnet, D., Bourgine, B., Dubois, D., Fargier, H., Come, B., Chiles, J.P., 2003. Hybrid approach for addressing uncertainty in risk assessments. Journal of Environmental Engineering, $129: 68-78$.

Guyonnet, D., Bellenfant, G., Bouc, O., 2008. Soft methods for treating uncertainties: applications in the field of environmental risks. Advances in Intelligent and Soft Computing, 48:16-26.

Kentel, E., Aral, M.M., 2005. 2D Monte Carlo versus 2D fuzzy Monte Carlo health risk assessment. Stochastic Environmental Research and Risk Assessment, 19:86-96

Klein, A. 2002. Gasification: An Alternative Process for Energy Recovery and Disposal of Municipal Solid Wastes. M.S. Thesis in Earth Resources Engineering, Columbia University.

Klir, G.J. \& Yuan, B. 1995. Fuzzy Sets and Fuzzy Logic: Theory and Applications. Upper Saddle River, NJ: PrenticeHall.

Kumar, V., Montse, M., Schuhmacher, M., Domingo, J.L. 2009. Partitioning total variance in risk assessment: Application to a municipal solid waste incinerator. Environmental Modelling \& Software, 24:247-261

Li, J., Huang, H.G., Zeng, G., Maqsood, I., Huang, Y. 2007. An integrated fuzzy-stochastic modelling approach for risk assessment of groundwater contamination. Journal of Environmental Management, 82: 177-188.

Lonati, G., Cernuschi, S., Giugliano, M. Grosso M. 2007. Health risk analysis of PCDD/F emissions from MSW incineration: comparison of probabilistic and deterministic approaches. Chemosphere, 67: S334-S343.

Porteous, A. 2005. Why energy from waste incineration ia an essential component of environmentally responsible waste management. Waste Management, 25: 451-459.

Schuhmacher, M., Meneses, M., Xifrò, A., Domingo J.L. 2001. The use of Monte-Carlo simulation techniques for risk assessment: study of a municipal waste incinerator. Chemosphere, 43: 787-799.

Sonnemann, G.W., Pla, Y., Schuhmacher, M., Castells, F. 2002. Framework for the uncertainty assessment in The Impact Pathway Analysis with an application on a ocal scale in Spain. Environment International, 28: 9-18.

Yamada, S., Shimizu, M., Miyoshi, F. 2004. Thermoselect Waste Gasification and Reforming Process. JFE technical report, 3: 20-24. 